\section{BMJ Open} Ophthalmology

\title{
Efficacy of vision-based treatments for children and teens with amblyopia: a systematic review and meta-analysis of randomised controlled trials
}

Taylor Adrian Brin (1D , ${ }^{1}$ Amy Chow, ${ }^{1}$ Caitlin Carter, ${ }^{2}$ Mark Oremus, ${ }^{3}$ William Bobier, ${ }^{1}$ Benjamin Thompson ${ }^{1,4,5}$

To cite: Brin TA, Chow A, Carter C, et al. Efficacy of vision-based treatments for children and teens with amblyopia: a systematic review and meta-analysis of randomised controlled trials. BMJ Open Ophthalmology 2021;6:e000657. doi:10.1136/ bmjophth-2020-000657

- Additional supplemental material is published online only. To view, please visit the journal online (http://dx.doi. org/10.1136/bmjophth-2020000657).

Received 10 November 2020 Revised 18 February 2021 Accepted 13 March 2021

Check for updates

(c) Author(s) (or their employer(s)) 2021. Re-use permitted under CC BY-NC. No commercial re-use. See rights and permissions. Published by BMJ.

${ }^{1}$ Optometry and Vision Science, University of Waterloo, Waterloo, Ontario, Canada

'Library, University of Waterloo, Waterloo, Ontario, Canada ${ }^{3}$ Public Health and Health Systems, University of Waterloo, Waterloo, Ontario, Canada ${ }^{4}$ Center for Eye and Vision Research, 17W Science Park, Hong Kong, Hong Kong ${ }^{5}$ Liggins Institute, University of Auckland, Auckland, New Zealand

\section{ABSTRACT}

Objective To identify differences in efficacy between vision-based treatments for improving visual acuity (VA) of the amblyopic eye in persons aged 4-17 years old.

Data sources Ovid Embase, PubMed (Medline), the Cochrane Library, Vision Cite and Scopus were systematically searched from 1975 to 17 June 2020. Methods Two independent reviewers screened search results for randomised controlled trials of vision-based amblyopia treatments that specified change in amblyopic eye VA (logMAR) as the primary outcome measure. Quality was assessed via risk of bias and GRADE (Grading of Recommendations, Assessment, Development, and Evaluations).

Results of the 3346 studies identified, 36 were included in a narrative synthesis. A random effects meta-analysis (five studies) compared the efficacy of binocular treatments versus patching: mean difference -0.03 logMAR; $95 \% \mathrm{Cl} 0.01$ to 0.04 ( $\mathrm{p}<0.001)$, favouring patching. An exploratory study-level regression (18 studies) showed no statistically significant differences between vision-based treatments and a reference group of 2-5hours of patching. Age, sample size and prerandomisation optical treatment were not statistically significantly associated with changes in amblyopic eye acuity. A network meta-analysis (26 studies) comparing vision-based treatments to patching 2-5hours found one statistically significant comparison, namely, the favouring of a combination of two treatment arms comparing combination and binocular treatments, against patching 2-5 hours: standard mean difference: $2.63 ; 95 \% \mathrm{Cl} 1.18$ to 4.09. However, this result was an indirect comparison calculated from a single study. A linear regression analysis (17 studies) found a significant relationship between adherence and effect size, but the model did not completely fit the data: regression coefficient 0.022 ; $95 \%$ Cl 0.004 to 0.040 ( $p=0.02$ ).

Conclusion We found no clinically relevant differences in treatment efficacy between the treatments included in this review. Adherence to the prescribed hours of treatment varied considerably and may have had an effect on treatment success.

\section{Key messages}

What is already known about this subject?

Previous meta-analyses or systematic reviews comparing patching to binocular treatments have found no difference or insufficient data to draw any conclusions.

- Adherence rates to amblyopia treatments range widely and can be quite poor.

What are the new findings?

All treatments assessed were not clinically different from 2 to 5 hours patching.

Adherence rates are low in many studies, which may affect treatment success.

\section{How might these results change the focus of} research or clinical practice?

> Our results suggest that clinicians have multiple treatment options that they can select based on the needs of their patients.

- Variability exists in the efficacy of various treatments in terms of improving amblyopic eye visual acuity.

Future studies are encouraged to use objective measures of adherence, where possible, to better understand the true effect of amblyopia treatments.

\section{INTRODUCTION}

Amblyopia is a neurodevelopmental visual disorder that affects between $0.34 \%$ and $3.9 \%$ of the population. ${ }^{12}$ Unilateral amblyopia is typically defined as visual acuity (VA) worse than 20/30 in an otherwise healthy eye, alongside a two-line interocular VA difference. However, visual deficits caused by amblyopia extend beyond reduced VA and encompass broader deficits such as impaired contrast sensitivity, stereopsis, spatial localisation and global form and motion perception. ${ }^{4-10}$ These deficits may adversely impact everyday tasks such as reading or playing sports. ${ }^{11-13}$ Amblyopia also limits career opportunities in fields such as military service, law enforcement, 
aviation and surgery, ${ }^{3}$ due to minimum standards of VA and binocularity in these professions.

Unilateral amblyopia results from abnormal visual experience early in life, typically caused by an eye misalignment (strabismus), a significant refractive difference between the eyes (anisometropia) or both (mixed). Deficits arise from impaired cortical processing of visual input from the eye that is chronically defocussed or misaligned. ${ }^{14}$ While the exact pathophysiology of amblyopia remains unknown, recent evidence suggests that it is a disorder of binocular vision where interocular suppression may play a key role in the resulting visual deficits. ${ }^{15}$

This systematic review considers vision-based amblyopia treatments that manipulate visual input to the brain, with the intention of changing cortical processing. Conventionally, vision-based amblyopia treatments targeting only the nonamblyopic fellow eye are referred to as monocular treatments. Examples include patching of the fellow eye and the use of atropine drops ${ }^{16}$ or Bangerter filters ${ }^{17}$ to reduce fellow eye image quality. These treatments have been shown to effectively improve amblyopic eye VA when treatment adherence is maintained..$^{15} 1718$ More recently, binocular approaches that rebalance the strength of visual input between the two eyes ${ }^{19}{ }^{20}$ have been developed to overcome interocular suppression and encourage simultaneous perception. ${ }^{21} 22$ Binocular treatments are designed to improve both amblyopic eye VA and binocular visual function. ${ }^{20} 23-28$

A number of randomised controlled trials (RCTs) over the past two decades have evaluated the efficacy of monocular (eg, patching, atropine and Bangerter filters) and binocular treatments for improving amblyopic eye VA. Comparisons of vision-based treatments for patients with amblyopia have been examined in systematic reviews comparing patching against atropine ${ }^{29-31}$ or binocular treatments against patching. ${ }^{32-34}$ Only one review $^{35}$ included a meta-analysis, which was limited to two studies and two treatments. In general, published systematic reviews and meta-analyses found no significant differences between the various vision-based amblyopia treatments. ${ }^{36}$

Treatment adherence, the time the participant spends engaged in the therapy, is a key factor that is often overlooked when assessing treatment efficacy. Poor adherence leads to reduced treatment efficacy. ${ }^{37}{ }^{38}$ Holmes $e t a l^{35}$ attributed the lack of a treatment effect from their binocular approach to extremely poor adherence, as opposed to the method of the treatment itself. That is, the participants simply were not as engaged as expected. Studies of patching reveal that self-reported adherence rates are variable, ranging from $49 \%$ to $87 \% .^{38}$ Therefore, adherence rates can be quite low for children undergoing various types of amblyopia treatments, and this must be considered when determining the true effect of any given treatment.

We conducted this systematic review and metaanalysis to assess the comparative efficacy of vision-based treatments for improving VA of the amblyopic eye. Furthermore, we were interested in how treatment effect size may be impacted by adherence. Our study includes a large sample of RCTs in our systematic review and metaanalysis, with a subanalysis of adherence rates.

\section{METHODS}

\section{Search strategy}

Preferred Reporting Items for Systematic Reviews and Meta-Analyses (PRISMA) guidelines were followed in conducting this review. ${ }^{39}$ The research question and literature search keywords were devised following consultation with a team of clinical and research experts (see online supplemental materials). We used the Population, Intervention, Comparator, Outcome, Time, and Setting (PICOTS) framework (Cochrane Handbook for Systematic Reviews of Interventions ${ }^{40}$ to specify the parameters of the research question, develop the literature search strategy and devise the eligibility criteria for inclusion of studies in the review (table 1).

An information specialist (CC) used the PICOTS to build a comprehensive search strategy for the following databases: PubMed (Medline), Ovid Embase, The Cochrane Library, Scopus and VisionCite. The initial search strategy was developed for PubMed (Medline) and the syntax and search terms were adapted to the other databases. Where available, controlled vocabulary such as medical subject headings was included in the search strategies. The database searches are updated as of 17 June 2020 and the search results were limited

\begin{tabular}{|c|c|}
\hline PICOTS & Criteria \\
\hline Population & $\begin{array}{l}\text { Patients with amblyopia aged } 4-17 \text { years old ( } \pm 1 \text { year, to either the upper or lower end of that spectrum, but } \\
\text { not both), caused by strabismus and/or anisometropia with no other ocular pathologies, mental illnesses, } \\
\text { learning disabilities and/or systemic diseases. } n>5 \text { participants in the study. }\end{array}$ \\
\hline Intervention & Vision-based treatment conducted in a randomised clinical trial. \\
\hline Comparator & Other types of vision-based treatments. \\
\hline Outcome & Change in visual acuity of the amblyopic eye from baseline (logMAR) as the primary outcome. \\
\hline Timing & Any duration. \\
\hline Setting & Any environment (clinical or at home) and any country. \\
\hline
\end{tabular}




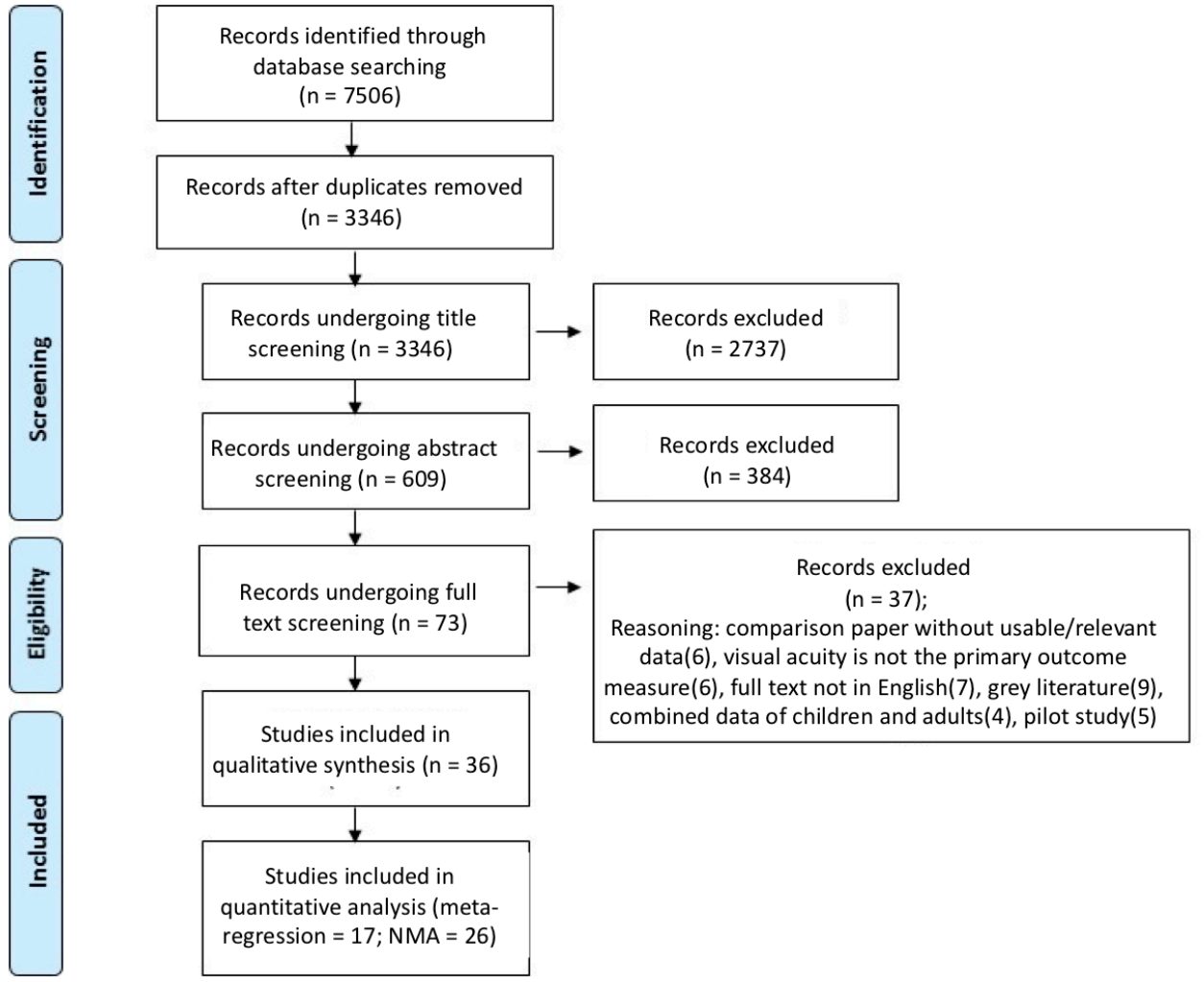

Figure 1 Flowchart of article screening and selection. NMA, network meta-analysis.

to English-language articles. The search strategy and PRISMA checklist are available as online supplemental materials.

\section{Screening}

Retrieved citations were imported into RefWorks (ProQuest LLC) for duplicate removal; remaining citations were transferred to DistillerSR (Evidence Partners) for screening by two independent reviewers (AC, TAB) at three levels: title, abstract and full text (figure 1). A third independent reviewer (WB) resolved discrepancies at the abstract and full-text levels. Citations generating discrepancies at title screening were advanced to abstract screening. Article eligibility criteria governing screening were:

Inclusion:

$\checkmark$ RCTs.

- Full-text published in English.

- Published between 1975 and 17 June 2020.

- Investigated one of the following vision-based treatments: patching or Bangerter filters, atropine, binocular treatments (any treatment using both eyes together, excluding optical treatment); combination treatments (any combined treatment that involved patching in addition to another intervention) or optical treatment.

- At least one group in the study included a vision-based treatment (eg, the other group could be a placebo).

Exclusion:
- Grey literature, conference abstracts, letters, commentaries, review articles or study designs other than RCTs or

- Only investigated treatments that could be categorised as placebos (eg, a monocular version of a video game as the control group for a binocular game) or that did not directly manipulate visual input to the brain (eg, acupuncture).

\section{Data extraction}

Two reviewers (AC, TAB) independently performed double entry data to extract the following information from each study: starting and final sample sizes, mean and SD of age in each group (or overall, if not available), treatment type, treatment dosage, mean and SD of change in VA of the amblyopic eye from baseline in $\log$ MAR, 95\% CIs of mean difference between treatments, study duration, setting (whether the treatment was prescribed for use at home or in-office) and treatment adherence rates.

\section{Risk of bias}

$\mathrm{AC}$ and $\mathrm{TAB}$ independently assessed the risk of bias (RoB) of the included RCTs at the study level using the Cochrane Risk of Bias 2 tool (22 August 2019 version). ${ }^{40}$ WB resolved all RoB disagreements. If information related to RoB was not reported, the authors of the study were contacted by e-mail for clarification. Some studies did not mask the outcome assessor, but the concern of it introducing bias was often mitigated through the use of well-validated and automated VA systems. Since poor 
adherence is a well-documented issue with patching, ${ }^{38}$ the risk of bias assessment included treatment adherence. For these studies, adherence was primarily based on participant reports.

To assess whether adherence affected the effect sizes (Hedges' $g$ ) of treatment comparisons, we regressed Hedges' $g$ onto the adherence rates for 26 studies that reported adherence data for all treatment and comparator groups.

\section{Meta-analysis}

We conducted a meta-analysis (five studies) comparing patching to binocular treatments. ${ }^{354-44}$ The inverse variance method, DerSimonian-Laird estimator for $\tau^{2}$, and a random effects model to obtain a pooled mean difference and $95 \%$ CI for the study-specific mean differences were used to carry out the meta-analysis. There was a high degree of heterogeneity between the studies, with $\mathrm{I}^{2}=80 \% ; \chi^{2}=19.74 \quad(\mathrm{p}<0.001)$, and $\tau^{2}=0.0017$. We used the 'meta' package in R V.4.0.2 (The R Foundation for Statistical Computing, Vienna, Austria) to conduct the meta-analyses. GRADEpro (Grading of Recommendations, Assessment, Development, and Evaluations) software (Hamilton, ON: Evidence Prime) was used to evaluate the overall certainty of evidence.

\section{Study-level regression}

We conducted an exploratory regression analysis at the study level to examine the relative effect of different treatments on VA. The dependent variable was the treatment-specific improvement in mean amblyopic eye VA from baseline to the end of the trial, as reported in each RCT. The unit of measuring VA was the logarithm of the minimum angle of resolution (logMAR). We included patching 2-5 hours, patching 6-11 hours, patching 12 or more hours, atropine, binocular treatment, combination treatment and intermittent patching (30s on, 30s off, using specialised glasses) in the regression analysis. Atropine, binocular treatments and combination treatments did not have a sufficient number of studies to permit separation by dosage.

We modelled each treatment as a dummy variable and used patching 2-5 hours as the reference category. The regression coefficients represented the change in VA of the amblyopic eye for each treatment compared with patching 2-5 hours. Patching 2-5 hours was chosen as the reference because it was the most common treatment dosage employed across RCTs. ${ }^{45} 46$ We controlled for patient mean age (or median age if the RCT did not report mean age), sample size and whether participants were given optical treatment for four or more weeks prior to the start of the trial.

Since each RCT evaluated two treatments, we modelled 'study' as a group-level, random effects variable and fit a restricted maximum likelihood linear mixed model to the data. The other variables (age, sample size and whether spectacles were prescribed at least 4 weeks prior to the start of the trial) were treated as fixed effects. We used the 'Ime4' package in R V.4.0.2 to conduct the analysis.

\section{Network meta-analysis}

To infer relationships between a broader number of treatments beyond those that were directly investigated in head-to-head trials, we undertook a frequentist network meta-analysis (NMA). We used a random effects model to conduct the NMA and measured statistical heterogeneity using the $\mathrm{X}^{2}$ test and $\mathrm{I}^{2}$ statistic. For each direct treatment comparison, we extracted the treatment-specific mean changes in logMAR over follow-up and obtained a common effect size, namely, Hedges' $g$ (a type of standard mean difference (SMD)). Studies that were missing sufficient data to calculate Hedges' $g$ were excluded from the analysis. Patching treatments were separated into four categories based on the daily prescribed dosage. Combination of treatments was separated by daily prescribed dosage and whether the additional activities were performed at near or at distance. Three studies used a three-arm treatment design, with active therapies including two different binocular treatments ${ }^{47}$ or a combination treatment and binocular treatment. ${ }^{434}$ The active treatments were combined, and then the SMD was calculated for a combined active category and patching 2-5 hours.

Certainty of treatment efficacy was ranked using p scores, which are analogous to surface under the cumulative ranking curve scores. ${ }^{48}$ We generated plots to estimate the proportion of direct and indirect evidence contributing to each possible comparison, minimal parallelism and mean path length. Furthermore, we explored the possibility of publication bias using a comparison-adjusted funnel plot and Egger's test (see online supplemental materials). We used the 'esc', 'netmeta', and 'dmetar' packages in R V.4.0.2 to conduct the NMA.

\section{Patient and public involvement statement}

It was not feasible to involve patients or the public in the design, conduct, reporting or dissemination of this project, as it is a meta-analysis on research that has already been conducted.

\section{RESULTS}

Following duplicate removal, 3346 citations advanced to the screening phase. We ultimately included 36 RCTs $(1 \%)$ in the narrative synthesis. From this 36 , 5 RCTs (14\%) were included in the meta-analysis, 18 in the regression analysis $(50 \%)$ and 26 in the NMA $(72 \%)$. The $\kappa$ for the two screeners was 0.77 at the title and abstract levels (combined) and 1.00 at the full-text screening level.

\section{Narrative synthesis of included studies}

All types of vision-based treatments produced VA improvements ranging from $0.06 \log$ MAR to 0.48 $\log$ MAR, except for two studies (Pawar et al.; Lee et 


\section{Frequency of Vision Therapy Categories}

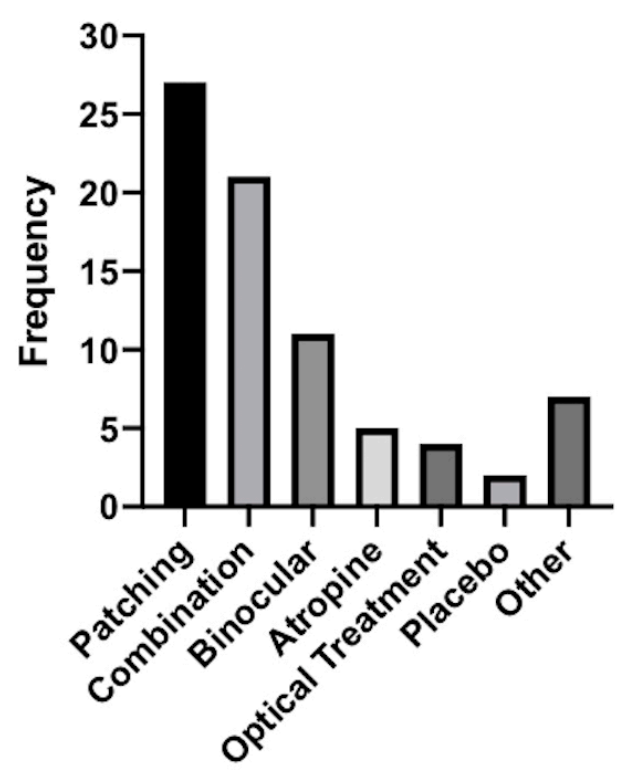

Figure 2 Frequency of vision-based treatments in the literature.

al.) in which VA declined after patching ${ }^{49}$ or patching combined with perceptual learning. ${ }^{44}$ While most treatments led to improved VA from baseline, less than half of the included RCTs $(n=17)$ reported clinically meaningful improvement, which is conventionally defined as a mean improvement in VA of $>2$ lines (or $0.2 \log$ MAR). ${ }^{1}$ The most common treatments to achieve this threshold were patching or Bangerter filters (14 conditions) and combination treatments (9). In only 5 of these 17 studies, the active treatment showed a statistically significant difference in amblyopic eye VA improvement from the control group. Therefore, it is rare for studies to show both clinical (an improvement of at least $0.2 \log \mathrm{MAR}$ ) and statistical significance.

Figure 2 shows the frequency with which each treatment category appeared in the 36 included RCTs, with patching being the most common therapy. Placebo treatments were the least common comparison, likely due to concerns over delaying treatment for young patients. The range of mean ages of participants in the included RCTs was 4.0-14.3 years. Only 10 RCTs had a mean age that was $>7$ years.

\section{Treatment adherence}

Of the 36 included studies, risk of bias was low in 17 and high in 12 (see online supplemental materials). The main reason for high risk of bias was poor adherence rates (seven studies). Adherence to amblyopia treatments was most commonly measured in the literature according to categories set by the Pediatric Eye Disease Investigator Group (PEDIG) ${ }^{50}$ PEDIG classifies adherence for individual study participants using a percentage score that is calculated by dividing the reported actual dose by the examiner's prescribed dose. These scores were grouped into four categories: 'excellent' (76\%-100\%), 'good' (51\%-75\%), 'fair' $(26 \%-50 \%)$ and 'poor' $(0 \%-25 \%)$. Using these four categories, PEDIG reports the number or percentage of patients in a treatment arm that achieves 'excellent' adherence.

Twenty-one of the 36 studies fully reported subjective adherence using the PEDIG classification standards. Over three-quarters of patients achieved 'excellent' adherence in only 10 studies. Six studies reported less than half of patients reporting excellent adherence, with the lowest adherence score being a study by Manh et al, wherein only $13 \%$ of patients reported excellent adherence. ${ }^{42}$ Given this variation, it was necessary to examine whether poor adherence influenced the published improvements in VA.

Figure 3 shows the linear regression line between Hedges' $g$ and adherence rates. When looking at the 17 studies that fully reported adherence rates, the linear regression was significant, demonstrating that treatments with high adherence rates showed larger effect sizes favouring the intervention treatment: regression coefficient $0.022 ; 95 \%$ CI 0.004 to $0.040 \quad(\mathrm{p}=0.020)$. However, the model does not fully explain the data. The regression line may exaggerate the relationship of adherence and effect size.

\section{Meta-analysis}

\section{Binocular treatment versus patching}

We performed a meta-analysis on five RCTs $^{35}{ }^{41-43}$ comparing the means of VA improvement for binocular treatments against patching. Figure 4 shows the difference between patching and binocular treatments, which was statistically significant at the $5 \%$ level $(-0.03 \operatorname{logMAR}$; $95 \%$ CI 0.01 to 0.04$)$. However, this difference is less than two letters and is not clinically significant. There was a high degree of heterogeneity between the studies, with $\mathrm{I}^{2}=80 \%$ and $\mathrm{X}_{4}^{2}=19.74(\mathrm{p}<0.001)$. The overall GRADE certainty of evidence for these five studies was assessed, finding an overall low certainty of evidence. This rating was due to serious concerns with inconsistency (high heterogeneity) and low precision (the wide CIs).

\section{Comparison of multiple vision-based treatments}

The exploratory regression comparing any treatment to patching 2-5hours contained 18 studies. None of the treatments showed a statistically significant difference relative to patching $2-5$ hours per day (see online supplemental materials). Furthermore, all treatments showed less than a one letter difference in VA compared with $2-5$ hours of patching. Sample size, spectacle use and mean (median) age were not associated with improvements in amblyopic eye VA from baseline in the included RCTs.

An NMA compared all treatments to patching 2-5 hours; the values in the Forest plot, therefore represent the SMD of the treatment in question versus patching 


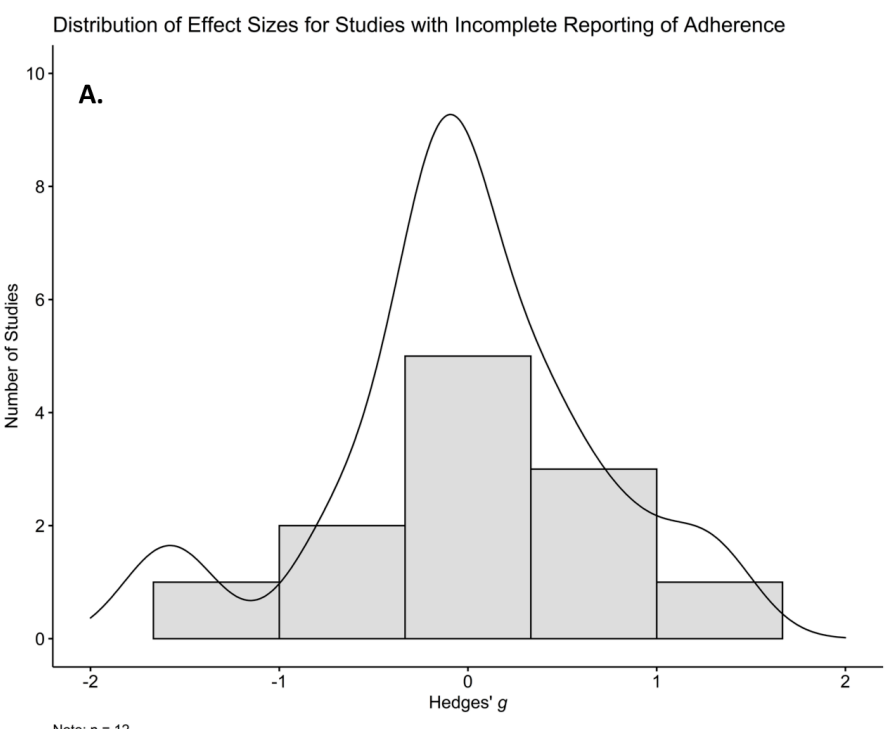

Note: $n=12$

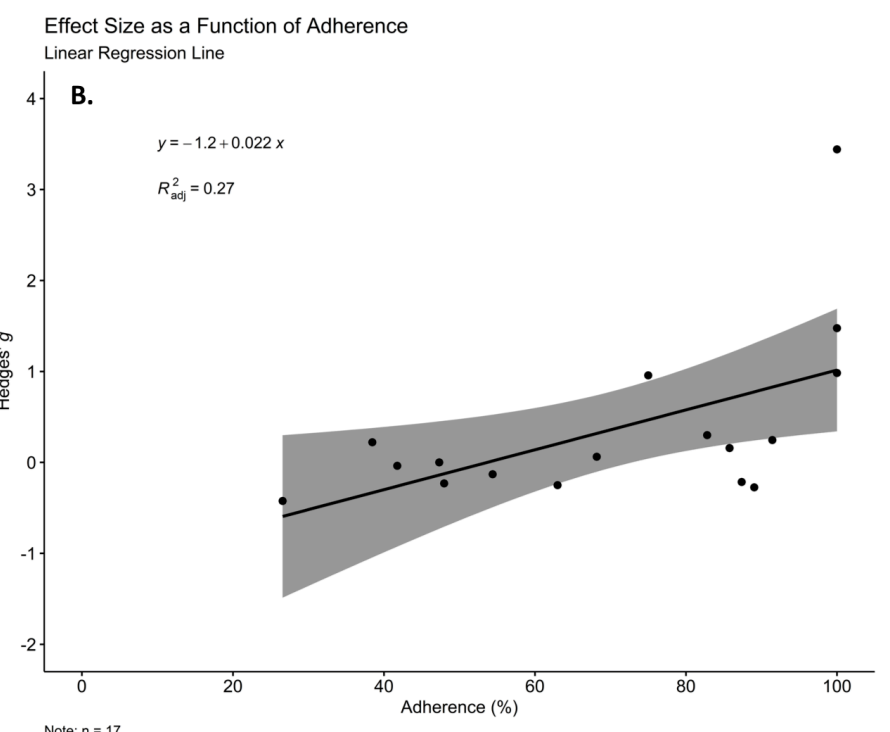

Note: $n=17$

Figure 3 Histogram (A) examined the Hedges' g of 12 studies with unreported or incomplete (eg, only reporting adherence rates for the active treatment) adherence data. The data for these studies do not appear to be biased. Scatterplot (B) shows the linear regression comparing effect size of each of the 17 studies as a function of reported adherence (with adherence defined as the percentage of patients achieving "excellent" adherence). Only studies with reported adherence data are included in this scatterplot.

2-5 hours. SMD $>0$ favours the treatment in question; SMD $<0$ favours patching $2-5$ hours.

The high level of heterogeneity $\left(\mathrm{I}^{2}=75.7 \%\right)$ in the NMA confirmed our decision to employ a random effects model. Twenty-six studies were included in the NMA, comparing 14 vision-based therapies to patching 2-5 hours and yielding 26 (direct and indirect) pairwise comparisons (figure 5). Most treatment comparisons involved patching or combination treatments.

The only comparison of SMD between groups that reached statistical significance was found between the combined binocular and combination group and patching 2-5hours with the combined binocular and combination group having a greater $\mathrm{SMD}(\mathrm{SMD}=2.63$, $95 \%$ CI 1.18 to 4.09 ). The p score for the combined binocular group was 0.9988 , indicating a high level of certainty for the efficacy of this treatment (see figure 6). However, the finding is from an indirect comparison, and only one of the included RCTs contains this type of therapy. The funnel plot did not show substantial evidence of asymmetry and Egger's test suggested that publication bias was not present $(\mathrm{p}=0.1151)$ (see online supplemental materials).
The results of the NMA should be interpreted with caution. Out of 105 total unique network estimates (treatment comparisons), only 20 contained some proportion of direct evidence (median proportion $=0.69$; $\mathrm{IQR}=0.60$ ). The remaining 85 estimates were based entirely on indirect evidence. For 90 of 105 estimates, the minimum number of independent paths contributing to the effect size estimate on an aggregated level (minimal parallelism) was 1 ; larger numbers of paths support more robust estimates, with the median number of paths being $2.1(\mathrm{IQR}=0.76)$ in the 15 comparisons with $>1$ minimum path. For mean path length, which characterises the degree of indirectness of an effect size estimate, values $>2$ indicate the need to interpret the estimate in question with caution. We found mean path lengths $>2$ in 80 of the 105 network estimates (plots available from the authors on request).

\section{DISCUSSION}

The objective of this systematic review and meta-analysis was to identify an optimal vision-based treatment for improving amblyopic eye VA in 4 to 17 year olds. Our analyses uncovered no clinically important differences

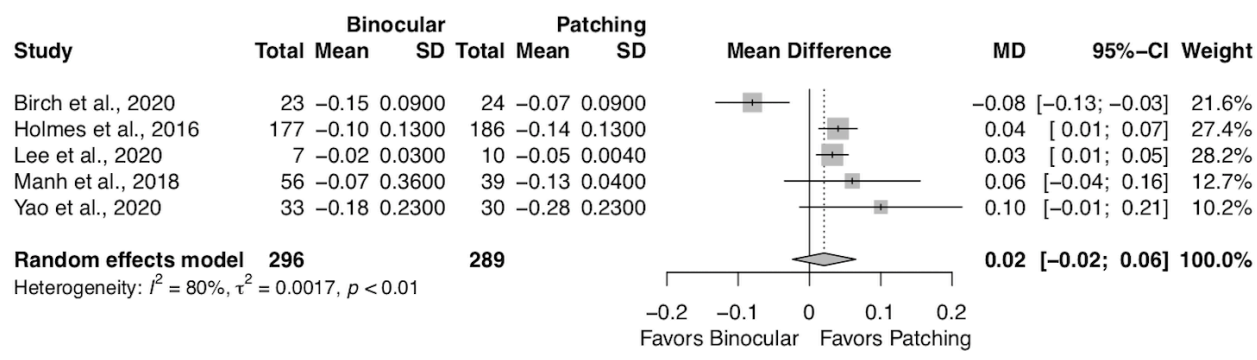

Figure 4 Forest plot comparing patching to binocular treatments. 


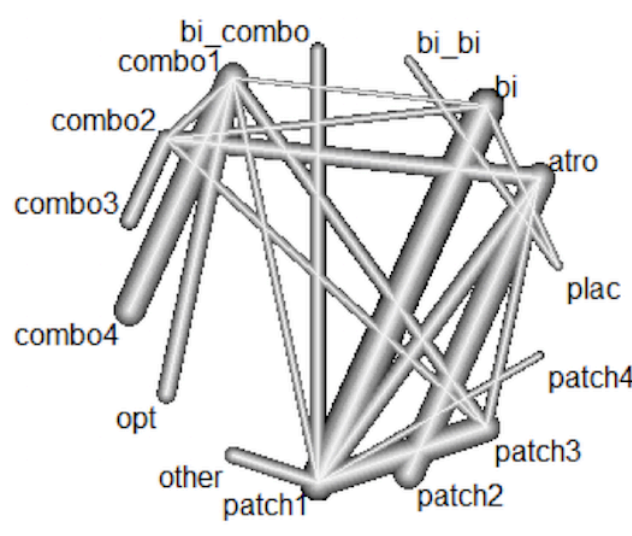

bi_combo: Combined (binocular and combination)

bi_bi: Combined (binocular and binocular)

bi: Binocular treatment

atro: Atropine

plac: Placebo

patch4: intermittent patching

patch3: Patching for $12+$ hours

patch2: Patching for 6-11 hours

patch1: Patching for 2-5 hours

other: non vision-based treatments

opt: Optical correction

combo4: patching and distance activities

combo3: patching and near activities for $12+$ hours

combo2: patching and near activities for $5-11$ hours

combo1: patching and near activities for 2-5 hours

Figure 5 Network graph of direct pairwise treatment comparisons. As the number of studies with a specific direct comparison increases, so does the thickness of the line.

between any of the treatments and patching 2-5 hours. Our adherence analysis revealed that poor adherence may be a factor in reducing treatment efficacy and may have affected our results. With high or unclear risk of bias in almost half the included RCTs, the findings of this review should be interpreted with caution.

Our results are similar to a previous NMA showing no significant difference between various amblyopia treatments, and that more research is needed. ${ }^{36}$ Several literature reviews have specifically compared the efficacy of binocular treatments to patching. A review by Pineles $e t$ al did not recommend the use of binocular treatments, ${ }^{33}$ while other systematic literature reviews concluded that more research was required before making any conclusions about binocular treatments. ${ }^{34}{ }^{51}$ More RCTs were available at the time of our literature search than these studies, but the overall strength of evidence for this comparison was low, which implies that further research is still required.

For the NMA, although it was not a significant result, we did not expect placebos to be considered more efficacious than patching $2-5$ hours. This result may have arisen because the comparison was indirect and only two studies used a placebo group. Furthermore, the adherence

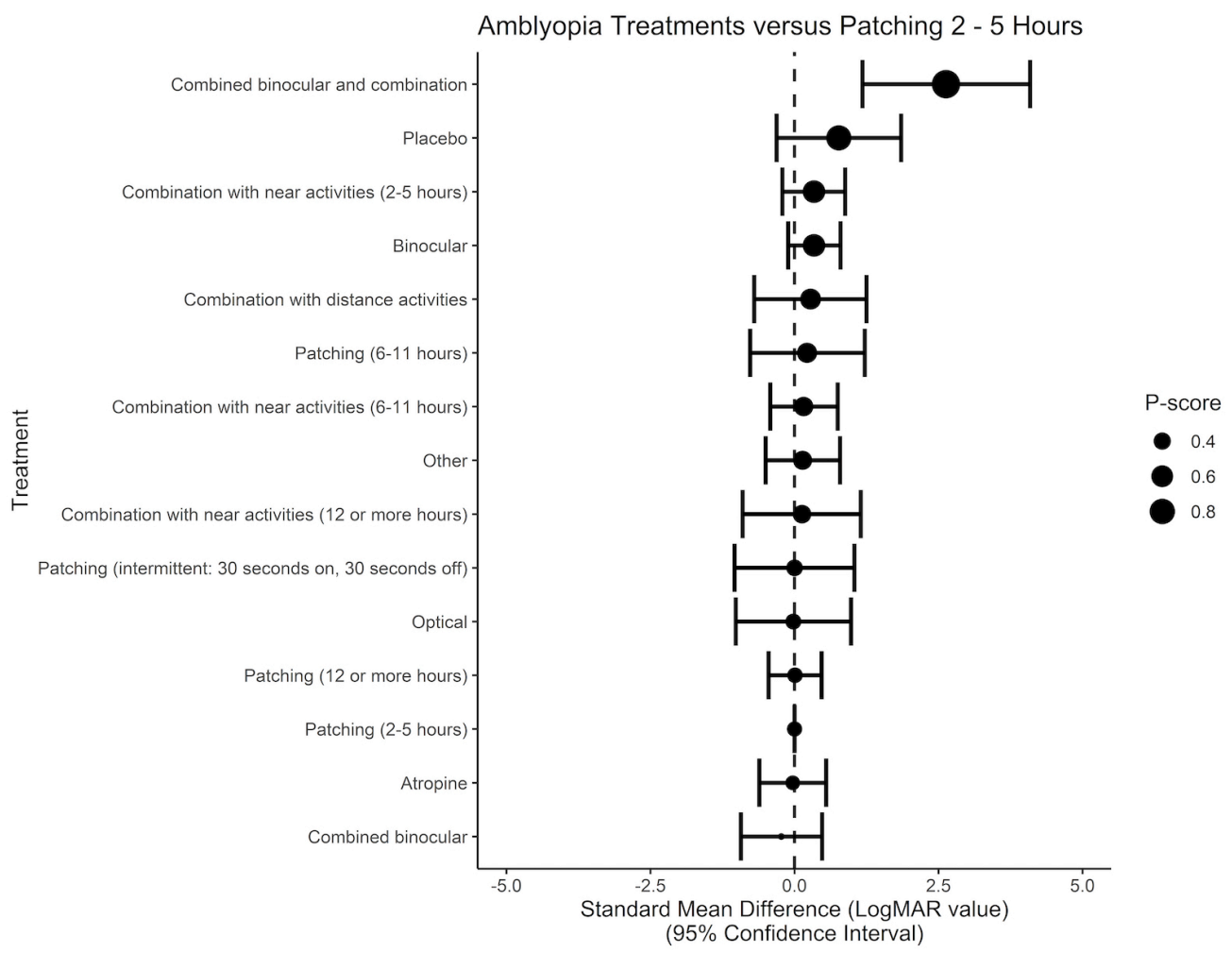

Figure 6 Forest plot of SMD and P-scores of treatments. The treatments are ranked from highest P-score (most efficacious) to lowest. SMD, standard mean difference. 
rate for the treatment group of one of the studies was very poor, ${ }^{52}$ which may explain why the placebo group is ranked as the second-best treatment in the NMA. Nonetheless, it is interesting to see how similar all visionbased treatments appear to be in terms of improving amblyopic eye VA. This implies that clinicians may have multiple treatment options. However, amblyopic eye VA improvements, in general, were small, as fewer than half of the studies reported an improvement greater than 2 LogMAR lines.

\section{Strengths and limitations of the literature}

One of the limitations of the literature is that the relatively small number of RCTs prevented us from conducting subanalyses by age or by dosage.

Our exploratory regression analysis showed that optical treatment prior to instituting another form of vision-based treatment was not significantly related to VA improvement. Since the studies that used optical treatment-prescribed spectacles to patients in every group, it was impossible to directly compare the effect of optical treatment to no optical treatment. Additionally, optical treatment durations were variable across many RCTs, with some employing a defined length of time (ranging widely from 4 to 18 weeks) and others waiting until the VA improvement reached a plateau.

Although our exploratory regression did not find an effect for age, it should be noted that $73 \%$ of the included RCTs featured a mean age of $<7$ years. It is possible we did not have a sufficiently wide enough range of ages to discern an effect.

Our meta-analyses revealed a high level of imprecision in the included studies, evidenced by wide CIs passing through the null value. A likely explanation for this variability is poor treatment adherence. It is critical to consider how low treatment adherence can negatively affect treatment efficacy. ${ }^{37}$ Poor adherence was the largest source of potential bias in studies, as identified in the RoB ratings. Of the studies that reported adherence rates, fewer than half had what would be considered good treatment adherence. It is also important to note that adherence data were almost entirely subjective. Many treatments took place at home, unsupervised by the experimenters and in uncontrolled environments. Adherence was reported by parents in the form of diaries or calendars. Subjective reports regularly overestimate adherence rates when compared with objective measures. ${ }^{38} 5354$ For example, Holmes et al prescribed a binocular video game treatment to be played at home and found that the average of parent-reported adherence was $66.7 \%$ of the total prescribed treatment time, while the game data revealed adherence to be $22.2 \% .^{35}$ Since the subjective adherence rates reported are likely higher than the actual adherence rate, this limits our ability to assess the true impact of adherence. However, these potentially inflated adherence rates were still poor, implying that the problem is more pronounced than what is reported here. Our linear regression showed a significant relationship between effect size and subjective adherence rates. However, the model does not fully explain the data, so this relationship may be exaggerated.

Where possible, robust objective measures should be used to ensure accuracy. Patching adherence can be objectively measured using occlusion dose monitors, which are modified eye patches that contain a battery and the ability to log data about the amount of time the patch is in contact with the skin around the eye. ${ }^{55}$ Some video game treatments can measure the amount of time a game is turned on or the number of log-ins, but there is no guarantee that the patient is actually looking at the screen while the game is powered on. The simplest option for ensuring adherence objectively is to administer treatment under supervised laboratory conditions, however cumbersome it may be for caregivers.

\section{Strengths and limitations of the review}

The major strength of this review is the comprehensive analysis of multiple vision-based therapies drawn from five different databases. ${ }^{356}$ We also included studies that could not be meta-analysed (due to insufficient data reported) in our systematic review to piece together a complete look at the relevant literature. Our results suggest that practitioners have a variety of equally effective treatments at their disposal and should be able to consider both patient and caregiver preferences in the management of amblyopia.

Another strength is the analysis of adherence rates. Previously, Li et al performed an NMA examining various vision-based treatments in patient with amblyopia and concluded that there was no clinically significant difference in the efficacy of these treatments. ${ }^{36}$ However, this study did not assess adherence rates, which we found to greatly impact the risk of bias rating. The goal of our adherence analysis was to control for adherence as much as possible when assessing treatment efficacy.

\section{CONCLUSION}

Vision-based treatments for amblyopia produce improvements in amblyopic eye VA for patients aged 4 to 17 years, but these improvements are not clinically significantly different from 2 to 5 hours of patching. Adherence must be considered when interpreting this result because many studies had poor or unreported adherence. One critical factor to consider for future studies is objective adherence monitoring, which may explain low treatment effects and high variability in a number of studies.

New vision-based treatments-such as binocular games-continue to be developed ${ }^{19}$ and may change the landscape of available treatment options for clinicians in 5-10 years time. It is imperative that the literature continues to be surveyed as new studies arise and our understanding of amblyopia evolves.

Acknowledgements The support of the Eye Health Council of Ontario is gratefully acknowledged. The selection of the initial bibliographic search terms was overseen by a Research Committee. Their contribution is gratefully acknowledged and a listing of its membership can be found in the supplementary material. 
Contributors All authors contributed to this project. The responsibilities were as follows: TAB: literature screening, data analysis, writing manuscript, editing manuscript. AC: literature screening, editing manuscript. CC: forming search strategy, literature search, editing manuscript. M0: data analysis, consultation, editing manuscript. WB: project inception, developing search strategy, editing manuscript, guiding the project. BT: editing manuscript, guiding the project.

Funding This research project received funding from the Canadian Optometric Education Trust Fund (COETF), The Natural Sciences and Engineering Research Council of Canada (NSERC), and the Canadian Institutes of Health Research (CIHR; grant 156174).

Competing interests BT holds two patents for a binocular treatment of amblyopia included in this review.

Patient consent for publication Not required.

Ethics approval The University of Waterloo Research Ethics Committee states that the secondary use of publicly available literature or other information does not require a review.

Provenance and peer review Not commissioned; externally peer reviewed.

Data availability statement Data are available upon request.

Supplemental material This content has been supplied by the author(s). It has not been vetted by BMJ Publishing Group Limited (BMJ) and may not have been peer-reviewed. Any opinions or recommendations discussed are solely those of the author(s) and are not endorsed by BMJ. BMJ disclaims all liability and responsibility arising from any reliance placed on the content. Where the content includes any translated material, BMJ does not warrant the accuracy and reliability of the translations (including but not limited to local regulations, clinical guidelines, terminology, drug names and drug dosages), and is not responsible for any error and/or omissions arising from translation and adaptation or otherwise.

Open access This is an open access article distributed in accordance with the Creative Commons Attribution Non Commercial (CC BY-NC 4.0) license, which permits others to distribute, remix, adapt, build upon this work non-commercially, and license their derivative works on different terms, provided the original work is properly cited, appropriate credit is given, any changes made indicated, and the use is non-commercial. See: http://creativecommons.org/licenses/by-nc/4.0/.

ORCID iD

Taylor Adrian Brin http://orcid.org/0000-0001-7749-5759

\section{REFERENCES}

1 Chia A, Dirani M, Chan Y-H, et al. Prevalence of amblyopia and strabismus in young singaporean Chinese children. Invest Ophthalmol Vis Sci 2010;51:3411-7.

2 Fu Z, Hong H, Su Z, et al. Global prevalence of amblyopia and disease burden projections through 2040: a systematic review and meta-analysis. Br J Ophthalmol 2020;104:1164-70.

3 Wallace DK, Repka MX, Lee KA, et al. Amblyopia preferred practice Pattern $®$. Ophthalmology 2018;125:P105-42.

4 Levi DM, Harwerth RS. Spatio-Temporal interactions in anisometropic and strabismic amblyopia. Invest Ophthalmol Vis Sci 1977;16:90-5.

5 Hess RF, Howell ER. The threshold contrast sensitivity function in strabismic amblyopia: evidence for a two type classification. Vision Res 1977;17:1049-55.

6 Levi DM, Waugh SJ, Beard BL. Spatial scale shifts in amblyopia. Vision Res 1994:34:3315-33.

7 Abrahamsson M, Sjöstrand J. Contrast sensitivity and acuity relationship in strabismic and anisometropic amblyopia. $\mathrm{Br} J$ Ophthalmol 1988;72:44-9.

8 Hess RF, Wang YZ, Demanins R, et al. A deficit in strabismic amblyopia for global shape detection. Vision Res 1999;39:901-14.

9 Aaen-Stockdale C, Hess RF. The amblyopic deficit for global motion is spatial scale invariant. Vision Res 2008;48:1965-71.

10 Hamm LM, Black J, Dai S, et al. Global processing in amblyopia: a review. Front Psychol 2014;5:583.

11 Kelly KR, Jost RM, De La Cruz A, et al. Amblyopic children read more slowly than controls under natural, binocular reading conditions. J Aapos 2015;19:515-20.

12 Birch EE, Castañeda YS, Cheng-Patel CS, et al. Self-perception in children aged 3 to 7 years with amblyopia and its association with deficits in vision and fine motor skills. JAMA Ophthalmol 2019:137:499-506.
13 Birch EE, Castañeda YS, Cheng-Patel CS, et al. Self-perception of school-aged children with amblyopia and its association with reading speed and motor skills. JAMA Ophthalmol 2019;137:167-74.

14 Barnes GR, Hess RF, Dumoulin SO, et al. The cortical deficit in humans with strabismic amblyopia. J Physiol 2001;533:281-97.

15 Scheiman MM, Hertle RW, Kraker RT, et al. Patching vs atropine to treat amblyopia in children aged 7 to 12 years: a randomized trial. Arch Ophthalmol 2008;126:1634-42.

16 Pediatric Eye Disease Investigator Group. The course of moderate amblyopia treated with atropine in children: experience of the amblyopia treatment study. Am J Ophthalmol 2003;136:630-9.

17 Pediatric Eye Disease Investigator Group Writing Committee, Rutstein RP, Quinn GE, et al. A randomized trial comparing Bangerter filters and patching for the treatment of moderate amblyopia in children. Ophthalmology 2010;117:998-1004-1006.

18 Repka MX, Cotter SA, Beck RW, et al. A randomized trial of atropine regimens for treatment of moderate amblyopia in children. Ophthalmology 2004;111:2076-85.

19 Pediatric eye disease investigator G, Holmes JM, Manny re, et al. A randomized trial of binocular dig rush game treatment for amblyopia in children aged 7 to 12 years. Ophthalmology 2019;126:456-66.

20 Li J, Thompson B, Deng D, et al. Dichoptic training enables the adult amblyopic brain to learn. Curr Biol 2013;23:R308-9.

21 Hess RF, Mansouri B, Thompson B. A new binocular approach to the treatment of amblyopia in adults well beyond the critical period of visual development. Restor Neurol Neurosci 2010;28:793-802.

22 Hess RF, Thompson B. Amblyopia and the binocular approach to its therapy. Vision Res 2015;114:4-16.

23 To L, Thompson B, Blum JR, et al. A game platform for treatment of amblyopia. IEEE Trans Neural Syst Rehabil Eng 2011;19:280-9.

24 Hess RF, Thompson B, Black JM, et al. An iPod treatment of amblyopia: an updated binocular approach. Optometry 2012;83:87-94

25 Kelly KR, Jost RM, Wang Y-Z, et al. Improved binocular outcomes following binocular treatment for childhood amblyopia. Invest Ophthalmol Vis Sci 2018;59:1221-8.

26 Bossi M, Tailor VK, Anderson EJ, et al. Binocular therapy for childhood amblyopia improves vision without breaking Interocular suppression. Invest Ophthalmol Vis Sci 2017;58:3031-43.

27 Vedamurthy I, Nahum M, Huang SJ, et al. A dichoptic custom-made action video game as a treatment for adult amblyopia. Vision Res 2015;114:173-87.

28 Mitchell DE, Duffy KR. The case from animal studies for balanced binocular treatment strategies for human amblyopia. Ophthalmic Physiol Opt 2014;34:129-45.

29 Li T, Shotton K, Cochrane Eyes and Vision Group. Conventional occlusion versus pharmacologic penalization for amblyopia. Cochrane Database Syst Rev 2009;81:CD006460.

$30 \mathrm{Li} \mathrm{T}$, Qureshi R, Taylor K. Conventional occlusion versus pharmacologic penalization for amblyopia. Cochrane Database Syst Rev 2019;8:CD006460.

31 Osborne DC, Greenhalgh KM, Evans MJE, et al. Atropine Penalization versus occlusion therapies for unilateral amblyopia after the critical period of visual development: a systematic review. Ophthalmol Ther 2018;7:323-32.

32 Tailor V, Bossi M, Bunce C, et al. Binocular versus standard occlusion or blurring treatment for unilateral amblyopia in children aged three to eight years. Cochrane Database Syst Rev 2015;8:CD011347.

33 Pineles SL, Aakalu VK, Hutchinson AK, et al. Binocular treatment of amblyopia: a report by the American Academy of ophthalmology. Ophthalmology 2020;127:261-72.

34 Hernández-Rodríguez CJ, Piñero DP. Active vision therapy for anisometropic amblyopia in children: a systematic review. $J$ Ophthalmol 2020;2020:1-9.

35 Holmes JM, Manh VM, Lazar EL, et al. Effect of a binocular iPad game vs part-time patching in children aged 5 to 12 years with amblyopia: a randomized clinical trial. JAMA Ophthalmol 2016;134:1391-400.

36 Li Y, Sun H, Zhu X, et al. Efficacy of interventions for amblyopia: a systematic review and network meta-analysis. BMC Ophthalmol 2020;20:203.

37 Woodruff G, Hiscox F, Thompson JR, et al. Factors affecting the outcome of children treated for amblyopia. Eye 1994;8:627-31.

38 Vagge A, Nelson LB. Compliance with the prescribed occlusion treatment for amblyopia. Curr Opin Ophthalmol 2017;28:454-9.

39 Liberati A, Altman DG, Tetzlaff J, et al. The PRISMA statement for reporting systematic reviews and meta-analyses of studies that evaluate healthcare interventions: explanation and elaboration. BMJ 2009;339:b2700. 
40 Higgins JPT TJ, Chandler J, Cumpston M. Cochrane Handbook for systematic reviews of interventions version 6.1, 2020. Available: www.training.cochrane.org/handbook

41 Kelly KR, Jost RM, Dao L, et al. Binocular iPad game vs patching for treatment of amblyopia in children: a randomized clinical trial. JAMA Ophthalmol 2016;134:1402-8.

42 Manh VM, Holmes JM, Lazar EL, et al. A randomized trial of a binocular iPad game versus part-time patching in children aged 13 to 16 years with amblyopia. Am J Ophthalmol 2018;186:104-15.

43 Yao J, Moon H-W, Qu X. Binocular game versus part-time patching for treatment of anisometropic amblyopia in Chinese children: a randomised clinical trial. Br J Ophthalmol 2020;104:369-75.

44 Lee YH, Maniglia M, Velez F, et al. Short-Term perceptual learning game does not improve Patching-Resistant amblyopia in older children. J Pediatr Ophthalmol Strabismus 2020;57:176-84.

45 Yazdani N, Sadeghi R, Momeni-Moghaddam H, et al. Part-time versus full-time occlusion therapy for treatment of amblyopia: A meta-analysis. J Curr Ophthalmol 2017;29:76-84.

46 Wallace DK. Pediatric eye disease investigator G, Edwards Ar, et al. A randomized trial to evaluate 2 hours of daily patching for strabismic and anisometropic amblyopia in children. Ophthalmology 2006;113:904-12.

47 Herbison N, MacKeith D, Vivian A, et al. Randomised controlled trial of video clips and interactive games to improve vision in children with amblyopia using the I-BiT system. Br J Ophthalmol 2016:100:1511-6.
48 Rücker G, Schwarzer G. Ranking treatments in frequentist network meta-analysis works without resampling methods. BMC Med Res Methodol 2015;15:58.

49 Pawar PV, Mumbare SS, Patil MS, et al. Effectiveness of the addition of citicoline to patching in the treatment of amblyopia around visual maturity: a randomized controlled trial. Indian J Ophthalmol 2014;62:124-9.

50 Pediatric Eye Disease Investigator Group.. A randomized trial of atropine vs. patching for treatment of moderate amblyopia in children. Arch Ophthalmol 2002;120:268-78.

51 Tsirlin I, Colpa L, Goltz HC, et al. Behavioral training as new treatment for adult amblyopia: a meta-analysis and systematic review. Invest Ophthalmol Vis Sci 2015;56:4061-75.

52 Gao TY, Guo CX, Babu RJ, et al. Effectiveness of a binocular video game vs placebo video game for improving visual functions in older children, teenagers, and adults with amblyopia: a randomized clinical trial. JAMA Ophthalmol 2018;136:172-81.

53 Wang J. Compliance and patching and atropine amblyopia treatments. Vision Res 2015;114:31-40.

54 Stewart CE, Stephens DA, Fielder AR, et al. Objectively monitored patching regimens for treatment of amblyopia: randomised trial. BMJ 2007;335:707-11.

55 Fronius M, Cirina L, Ackermann $\mathrm{H}$, et al. Efficiency of electronically monitored amblyopia treatment between 5 and 16 years of age: new insight into declining susceptibility of the visual system. Vision Res 2014;103:11-19.

56 Guyatt GH, Oxman AD, Kunz R, et al. Going from evidence to recommendations. BMJ 2008;336:1049-51. 